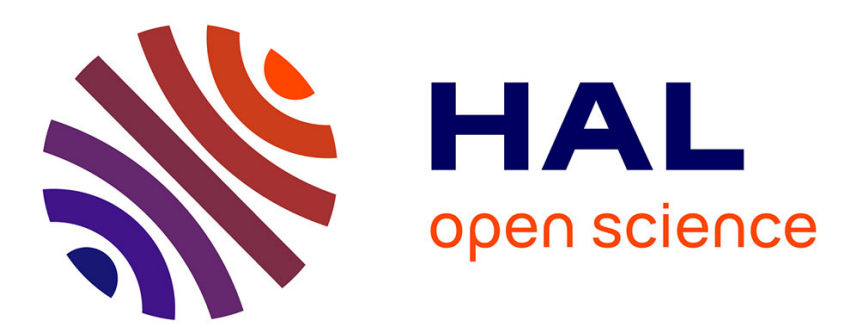

\title{
Appareil de mesure du module d'élasticité et du frottement intérieur en flexion, à basse fréquence, sous vide entre 20 et $800{ }^{\circ} \mathrm{C}$
}

J. Woirgard, J.-P. Amirault, H. Chaumet, J. de Fouquet

\section{- To cite this version:}

J. Woirgard, J.-P. Amirault, H. Chaumet, J. de Fouquet. Appareil de mesure du module d'élasticité et du frottement intérieur en flexion, à basse fréquence, sous vide entre 20 et $800{ }^{\circ} \mathrm{C}$. Revue de Physique Appliquée, 1971, 6 (3), pp.355-359. 10.1051/rphysap:0197100603035500 . jpa-00243555

\section{HAL Id: jpa-00243555 https://hal.science/jpa-00243555}

Submitted on 1 Jan 1971

HAL is a multi-disciplinary open access archive for the deposit and dissemination of scientific research documents, whether they are published or not. The documents may come from teaching and research institutions in France or abroad, or from public or private research centers.
L'archive ouverte pluridisciplinaire HAL, est destinée au dépôt et à la diffusion de documents scientifiques de niveau recherche, publiés ou non, émanant des établissements d'enseignement et de recherche français ou étrangers, des laboratoires publics ou privés. 


\title{
APPAREIL DE MESURE DU MODULE D'ÉLASTICITÉ ET DU FROTTEMENT INTÉRIEUR EN FLEXION, A BASSE FRÉQUENCE, SOUS VIDE ENTRE 20 ET $800{ }^{\circ} \mathrm{C}$
}

\author{
J. WOIRGARD, J.-P. AMIRAUlT, H. CHAUMET et J. de FOUQUET (*)
}

(*) Laboratoire de Mécanique et Physique des matériaux, rue Guillaume-VII 86, Poitiers (E. R. A.-C. N. R. S. no 123)

(Reģu le 25 mars 1971)

\begin{abstract}
Résumé. - Un appareil de mesure du module d'élasticité et du frottement intérieur a été réalisé, afin d'étudier en flexion et à basse fréquence les effets d'hystérésis et de relaxation des métaux. Cet appareil permet d'utiliser des échantillons en forme de lames de rigidité relativement élevée. La mesure du frottement intérieur se fait en oscillations libres. Des capteurs inductifs, sans contact, permettent de détecter des oscillations de flexion correspondant à des déformations maximales de l'éprouvette $\left(\varepsilon_{\max }\right)$ comprises entre $10^{-7}$ et $10^{-5}$, à des fréquences allant de $0,2 \mathrm{à} 10 \mathrm{~Hz}$, entre l'ambiante et $800^{\circ} \mathrm{C}$, et sous un vide de quelque $10^{-6}$ torr.

Abstract. - An apparatus allowing the measurement in flexion, at low frequencies, of modulus and internal friction related to hysteresis and relaxation phenomena in metals has been developed. It is possible to use specimen with relatively high rigidity. The internal friction measurements are performed by the free decay method. The flexural oscillations are detected without contact by inductive transducers. The outer fiber strain range available is from $10^{-7}$ to $10^{-5}$ and the frequency range 0.2 to $10 \mathrm{~Hz}$, between room temperature and $800^{\circ} \mathrm{C}$, under a vacuum of $10^{-6}$ torr.
\end{abstract}

Introduction. - Les méthodes utilisées pour étudier le frottement intérieur des métaux à faible fréquence (de l'ordre du $\mathrm{Hz}$ ) font généralement appel au pendule de torsion : pendule simple de Coulomb, pendule de Ké simple ou inversé, pendule composé de Collette [1], [2], [3]. Les éprouvettes sont soit des fils de section circulaire, de diamètre inférieur à $2 \mathrm{~mm}$, soit des lames d'épaisseur excédant rarement $1 \mathrm{~mm}$ afin d'éviter des volants d'inertie trop importants. Les gammes de fréquences ainsi obtenues ne dépassent pas, pour un même échantillon, quelques hertz au maximum; les enregistrements s'effectuent soit par mesure de la décroissance d'oscillations libres (méthodes optiques, cellules photoélectriques, photorésistances) soit à amplitude constante par mesure de l'énergie nécessaire pour entretenir le mouvement (bobines magnétiques, détection capacitive...) [4].

Le dispositif décrit ci-après a pour objet l'étude, à des fréquences faibles, mais pouvant varier entre 0,2 et $10 \mathrm{~Hz}$, d'éprouvettes d'épaisseur plus élevée (jusqu'à $2,5 \mathrm{~mm}$ ) et sollicitées en flexion, ce qui permet en particulier l'utilisation d'éprouvettes bicristallines avec joint longitudinal.

Principe. - L'éprouvette se présente sous la forme d'une lame encastrée à l'extrémité inférieure et solidaire à sa partie supérieure d'un tube rigide vertical (Fig. 1) suspendu à deux fils très fins permettant d'éviter toute contrainte statique sur l'éprouvette, grâce à un système d'équilibrage.

Une force $F$ appliquée normalement à l'extrémité supérieure du tube (Fig. 1) crée un moment fléchis-

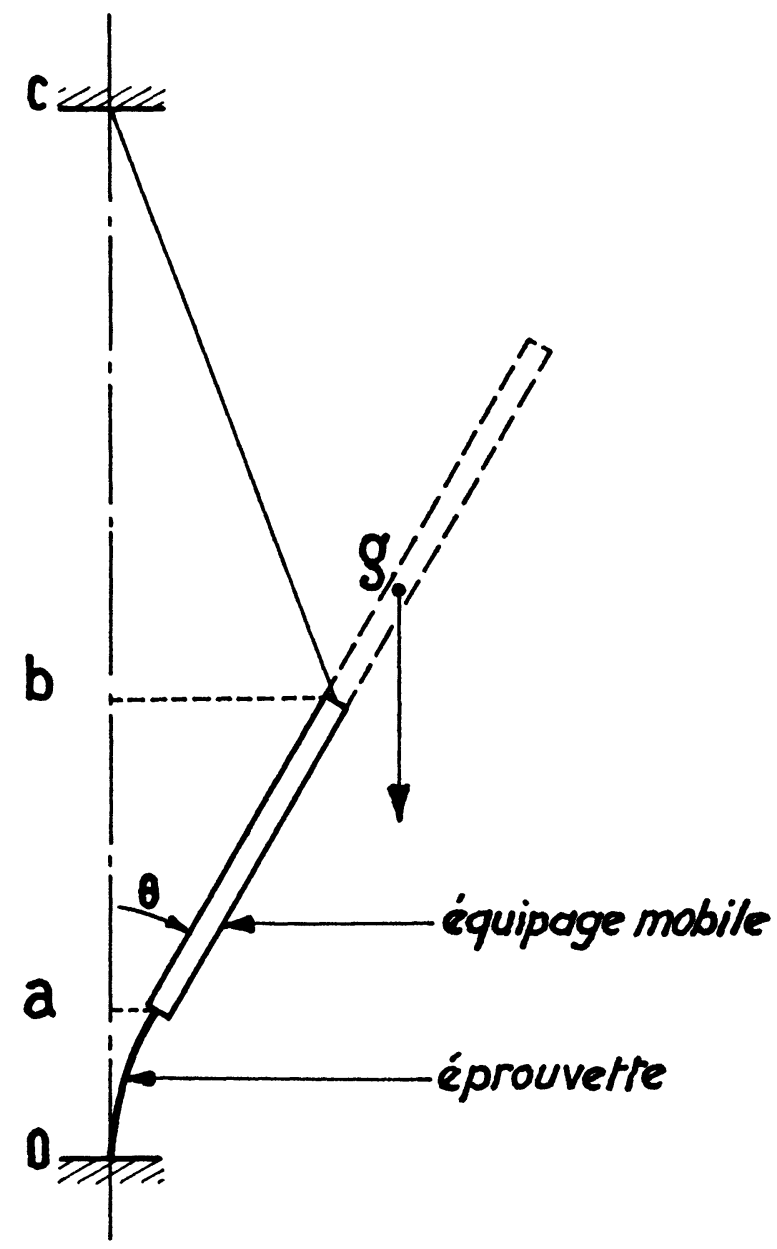

Fig. 1. - Principe de fonctionnement du pendule de flexion. 
sant dont la variation n'excède pas $16 \%$ le long de l'éprouvette, d'où un état de contraintes proche de la flexion simple. Les effets d'encastrement sont ainsi moins prononcés que dans la flexion encastrée et la sollicitation est plus homogène.

Une masse additionnelle cylindrique située dans le prolongement du tube et fixée à son extrémité supérieure (Fig. 1) permet de faire varier la fréquence dans un rapport voisin de 10 , ce qui dans le cas d'un pendule de torsion et pour des éprouvettes d'épaisseur comparable nécessiterait des volants d'inertie variant dans des proportions excessives.

Calculs. - Pour évaluer les efforts subis par l'éprouvette et les déformations correspondantes on suppose l'allongement de l'éprouvette négligeable devant celui des fils de suspension et on néglige également l'influence de l'effort tranchant ce qui revient à admettre une déformée de forme parabolique.

\section{NotaTions.}

\section{$b$ : largeur de l'éprouvette}

$e$ : épaisseur de l'éprouvette ;

$\overline{o a}=l_{1} \quad:$ longueur de l'éprouvette;

$I_{1}=b e^{3} / 12:$ moment de flexion de l'éprouvette ;

$E_{1}$ : module d'Young de l'éprouvette ;

$\overline{a b}=l_{2} \quad: b$ point d'attache des fils de suspension;

$\overline{a g}=L_{2} \quad: g$ centre de gravité de l'équipage mobile (avec ou sans masse additionnelle);

$P_{2}$ : poids de l'équipage mobile ;

$I_{2}$ : moment d'inertie de l'équipage mobile ;

$\overline{b c}=L_{3} \quad$ : longueur des fils de suspension;

$S_{3}$ : section des fils de suspension;

$E_{3}$ : module d'Young des fils de suspension.

Lorsque l'équipage mobile tourne d'un angle $\theta$ l'énergie acquise par le système se décompose en :

$$
W=W_{1}+W_{2}+W_{3}
$$

où $W_{1}$ désigne l'énergie élastique de l'éprouvette, $W_{2}$ l'énergie potentielle perdue par l'équipage mobile et $W_{3}$ l'énergie élastique des fils de suspension.

Compte tenu des hypothèses citées plus haut :

$$
\begin{aligned}
& W_{1}=\frac{1}{2} C_{1} \theta^{2} \\
& \text { avec } C_{1}=\frac{E_{1} I_{1}}{L} \\
& W_{2}=\frac{1}{2} C_{2} \theta^{2} \\
& \text { avec } C_{2}=-P_{2}\left(L_{2}+\frac{l_{1}}{3}\right) \\
& W_{3}=\frac{1}{2} C_{3} \theta^{2} \\
& \text { avec } C_{3}=P_{2} L_{2}\left(1+\frac{l_{1}}{3 l_{2}}+\frac{l_{2}+l_{1}}{L_{3}}\right)
\end{aligned}
$$

l'énergie totale s'écrira donc :

$$
W=\frac{1}{2}\left(C_{1}+C_{2}+C_{3}\right) \theta^{2}
$$

en désignant par $I_{2}$ le moment d'inertie de l'équipage mobile. La période d'oscillation $T$ est donnée par :

$$
T=2 \pi \sqrt{\frac{I_{2}}{C_{1}+C_{2}+C_{3}}} .
$$

Si l'on note $Q_{\mathrm{m}}^{-1}$ l'amortissement mesuré, $Q_{\mathrm{e}}^{-1}$ l'amortissement propre de l'éprouvette et $Q_{\mathrm{f}}^{-1}$ l'amortissement des fils de suspension:

$$
\begin{aligned}
Q_{\mathrm{m}}^{-1}=\frac{C_{1}}{C_{1}+C_{2}+C_{3}} \cdot Q_{\mathrm{e}}^{-1} & + \\
& +\frac{C_{3}}{C_{1}+C_{2}+C_{3}} \cdot Q_{\mathrm{f}}^{-1} .
\end{aligned}
$$

On peut constater que cette expression est équivalente à celle du pendule de torsion composé de Collette [3]. Il convient toutefois de remarquer que $C_{2}$ est ici négatif ce qui permet selon le signe de $C_{2}+C_{3}$ d'obtenir $Q_{\mathrm{m}}^{-1}$ inférieur, égal ou supérieur à $Q_{\mathrm{e}}^{-1}$.

1) Mesure sans masse additionnelle. - Dans ce cas $C_{2}+C_{3}>0$. Si l'on effectue une mesure avec une éprouvette de rigidité négligeable devant $C_{2}$ et $C_{3}$ on obtient la période

$$
T_{0}=2 \pi \sqrt{\frac{I_{2}}{C_{2}+C_{3}}} .
$$

Avec une éprouvette de rigidité $C_{1}$ donnant une période $T$ et possédant un amortissement $Q_{\mathrm{e}}^{-1}$; l'amortissement dû aux fils de suspension étant négligeable on a :

$$
Q_{\mathrm{m}}^{-1}=\left(1-\frac{T^{2}}{T_{0}^{2}}\right) \cdot Q_{\mathrm{e}}^{-1}
$$

et

$$
C_{1}=\left(C_{2}+C_{3}\right) \cdot\left(\frac{T_{0}^{2}}{T^{2}}-1\right)
$$

ce qui permet une mesure du module d'élasticité de l'éprouvette. Avec une éprouvette de $0,2 \mathrm{~mm}$ d'épaisseur nous avons trouvé $T_{0}=0,5 \mathrm{~s}$ ce qui donne pour une éprouvette d'aluminium de $2 \mathrm{~mm}$ d'épaisseur $(T=0,13 \mathrm{~s})$ :

$$
Q_{\mathrm{e}}^{-1}=1,07 Q_{\mathrm{m}}^{-1} \text {. }
$$

2) Mesure AVEC MASSE ADDitionnelle. - La masse additionnelle a été choisie de façon que $C_{2}+C_{3}=0$; on obtient alors:

$$
Q_{\mathrm{m}}^{-1}=Q_{\mathrm{e}}^{-1} \quad \text { et } \quad C_{1}=\frac{4 \pi^{2} I_{2}}{T^{2}}
$$

Réalisation. - La figure 2 donne une vue d'ensemble de l'appareil. On distingue l'enceinte à vide qui permet d'atteindre un vide de quelque $10^{-6}$ torr et le système de chauffage constitué par un four Adamel à trois enroulements et un enroulement 
supplémentaire situé à proximité du mors supérieur permettant de réduire le gradient sur l'éprouvette. Il est ainsi possible de chauffer de $20^{\circ} \mathrm{C}$ à $800^{\circ} \mathrm{C}$ avec un gradient sur l'éprouvette ne dépassant pas $2^{\circ} \mathrm{C}$.

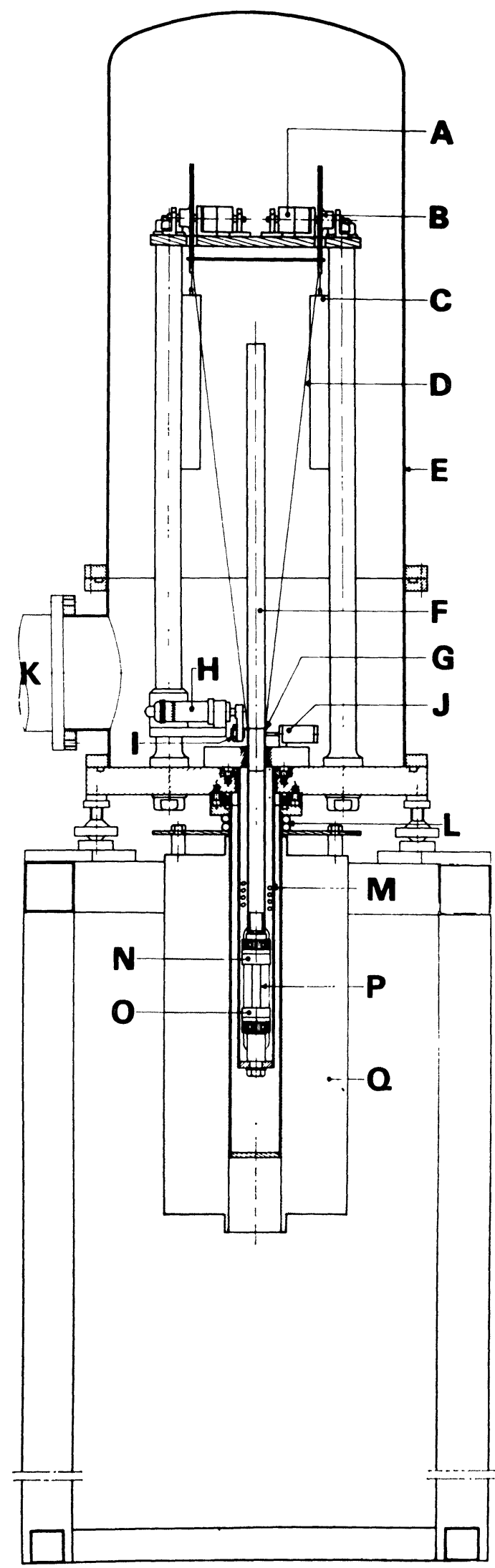

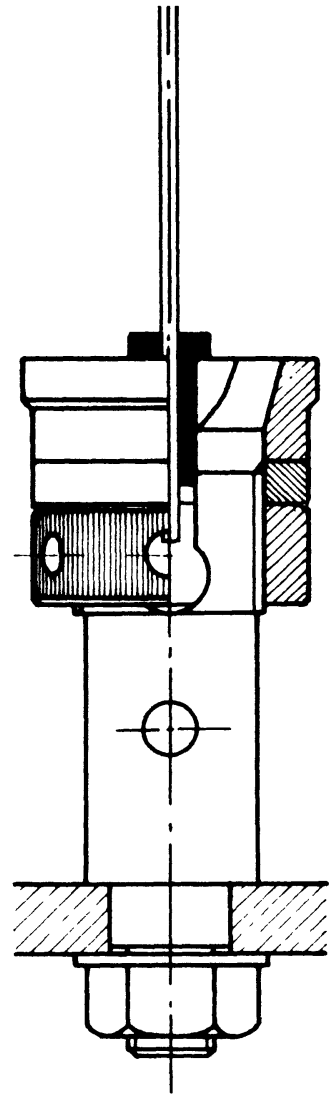

Fig. 3. - Schéma des mors de serrage de l'éprouvette.

- Les éprouvettes sont des lames de $50 \mathrm{~mm}$ de longueur utile. La largeur n'est pas fixée de façon impérative (on prend généralement $10 \mathrm{~mm}$ ) et l'épaisseur peut varier de 0,5 à $2,5 \mathrm{~mm}$.

- L'éprouvette, prise entre deux plaquettes de matériau présentant un faible amortissement propre est serrée par des mors en forme de pinces (Fig. 3).

- Un montage annexe permet d'assurer le positionnement de l'éprouvette par rapport aux mors et d'éviter tout risque d'écrouissage au cours du montage ou du démontage, ce qui est indispensable pour des essais sur monocristaux.

- Les deux fils d'équilibrage de l'équipage mobile (Fig. 2), sont disposés en $\mathrm{V}$ ce qui permet d'obtenir une rigidité en torsion suffisante pour éliminer totalement le mode de vibration de torsion.

Détection. - Pour mesurer les oscillations de flexion on détecte le mouvement de deux plaquettes ferromagnétiques fixées à l'extrémité du tube mobile au

FIG. 2. - Vue d'ensemble de l'appareil. A : Immobilisation des poulies ; B : Poulies ; C : Contrepoids ; D : Fils de suspension ; $\mathrm{E}$ : Enceinte à vide ; F : Masse additionnelle; $\mathrm{G}$ : Point d'attache des fils de suspension $; \mathrm{H}$ : Micromètre assurant le positionnement des capteurs ; I : Capteur de déplacement ; $J$ : Bobine d'excitation ; $\mathrm{K}$ : Arrivée de vide ; $\mathrm{L}$ : Refroidissement ; $M$ : Chauffage d'appoint ; $\mathbf{N}$ : Mors supérieur ; $\mathbf{O}$ : Mors inférieur ; $\mathbf{P}$ : Eprouvette ; $\mathrm{Q}:$ Four Adamel à trois enroulements. 
moyen de capteurs inductifs sans contact $\left({ }^{*}\right)$ alimentés par un pont de fréquence porteuse $5 \mathrm{kHz}$. Ce pont délivre une tension proportionnelle au déplacement des plaquettes pour des fréquences allant du continu à $1,5 \mathrm{kHz}$. Lorsque les capteurs sont situés suffisamment près de la surface de référence on obtient une sensibilité très élevée : pour une distance de $1 \mathrm{~mm}$ un déplacement de $\pm 1 \mu$ donne à la sortie du pont une tension de $\pm 2 \mathrm{~V}$. Grâce à ce dispositif il est théoriquement possible de travailler à des déformations maximales $\varepsilon_{\max }$, sur la fibre externe, de quelque $10^{-8}$; en pratique les vibrations parasites toujours difficiles à éliminer totalement limitent ce seuil à $10^{-7}$.

Excitation. - Pour exciter l'appareil et obtenir progressivement une amplitude de vibration donnée il est nécessaire d'envoyer des impulsions de même fréquence que le mouvement. On utilise pour cela le signal de sortie des capteurs, après filtrage, afin d'éliminer tous les parasites électriques (bande passante 0,1 à $20 \mathrm{~Hz}$ ). Ce signal est transformé en impulsions de hauteur et largeur réglables, pour tenir compte des variations d'amortissement c'est-à-dire des variations de l'énergie nécessaire à l'excitation, et envoyé à une bobine d'excitation à noyau plongeur situé à l'extrémité supérieure du tube mobile. Lorsque

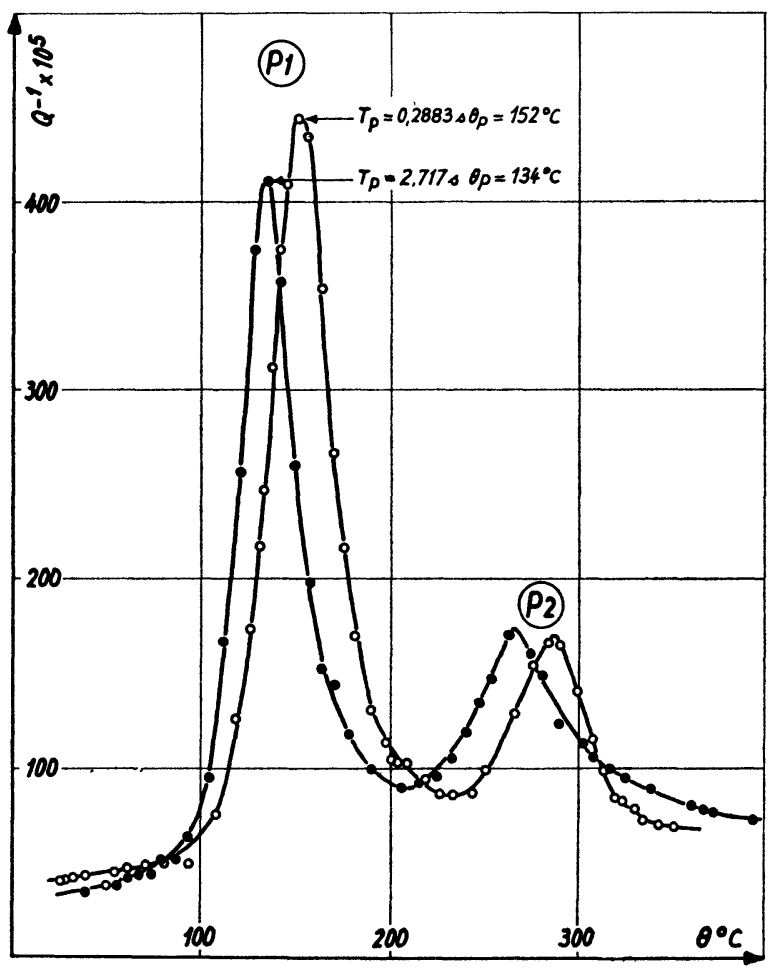

Fig. 4. - Spectres obtenus sur une éprouvette de Niobium de $1 \mathrm{~mm}$ d'épaisseur entre $20^{\circ} \mathrm{C}$ et $400^{\circ} \mathrm{C}$ avec un rapport de fréquence égal à 9,4 .

(*) Fabriqués, ainsi que le pont, par Hottinger Badwin Messtechnik.

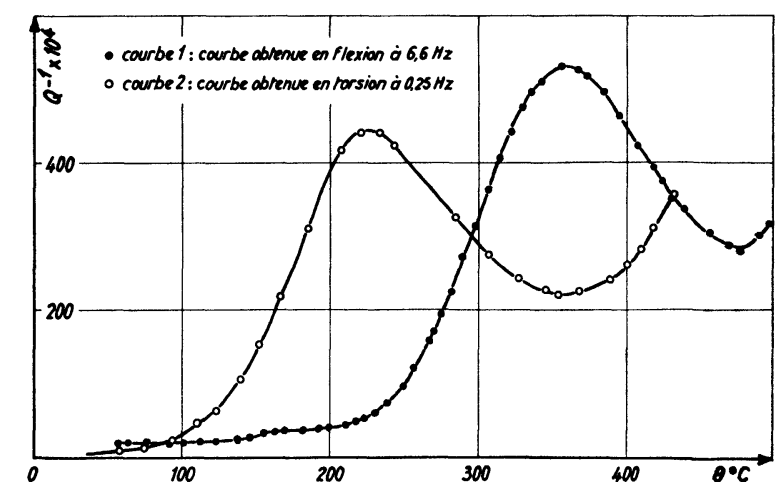

FIG. 5. - Spectres obtenus sur des éprouvettes d'Aluminium de pureté $5 \mathrm{~N}$ en torsion et en flexion. Diamètres de grains :

$0 \sim 1 \mathrm{~mm}$
$0 \sim 0,1 \mathrm{~mm}$.

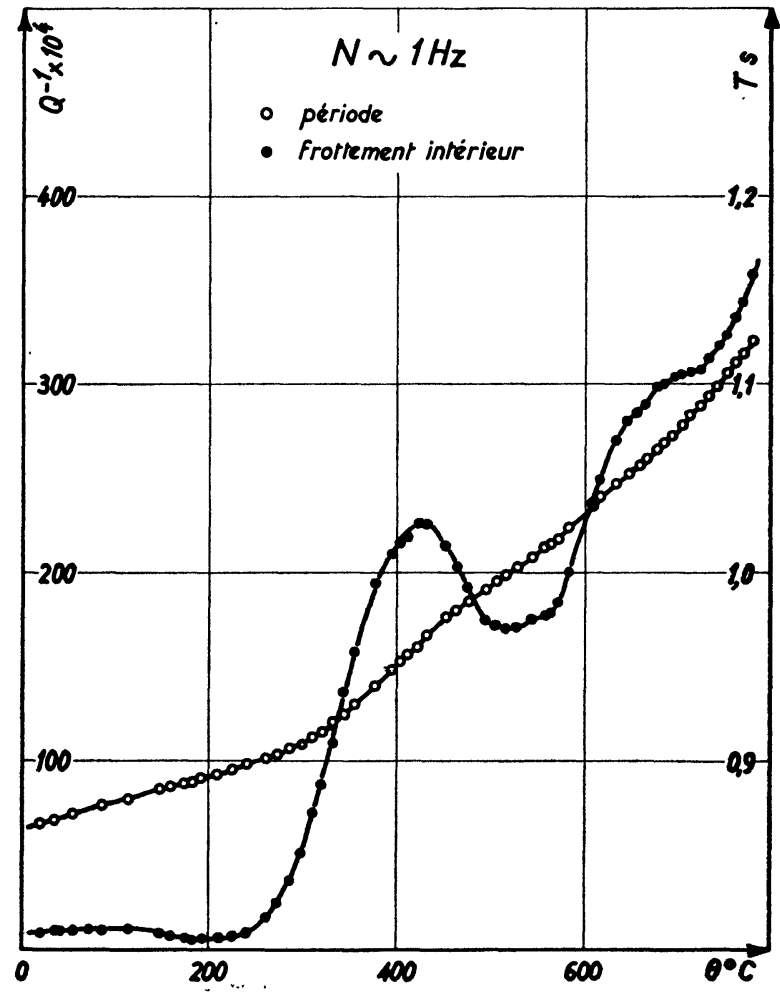

Fig. 6. - Spectre obtenu sur une éprouvette de Cuivre à $1 \mathbf{H z}$.

l'amplitude d'oscillation désirée est atteinte la mesure de l'amortissement se fait en oscillations libres. Le dispositif d'excitation réalisé permet en fait d'entretenir les oscillations ce qui constitue également une mesure précise de la période d'oscillation.

Enregistrement. - L'enregistrement des oscillations se fait au moyen d'un enregistreur à galvanomètre crayon et lampe à vapeurs de mercure ce qui permet son utilisation dans une gamme de fréquence élevée. 
Applications. - Nous avons effectué à titre de vérification différents essais sur des éprouvettes de Niobium, d'Aluminium et de Cuivre notamment.

La figure 4, courbe 1 montre le spectre obtenu sur une éprouvette de Niobium à une fréquence de $3,46 \mathrm{~Hz}$. On distingue le pic I dû à l'oxygène et le pic II dû au carbone et à l'azote. Un autre essai effectué à $0,36 \mathrm{~Hz}$ sur cette même éprouvette donne un déplacement du pic I de $18^{\circ} \mathrm{C}$ ce qui correspond bien à l'énergie d'activation de $27 \mathrm{kcal} / \mathrm{mole}$ admise pour ce pic.

La figure 5, courbe 1 représente le pic dû à la relaxation des joints de grain d'une éprouvette d'Aluminium de pureté $5 \mathrm{~N}$ pour une fréquence moyenne de $6,6 \mathrm{~Hz}$. On peut comparer cette courbe à celle obtenue (courbe 2) à l'aide d'un pendule de torsion composé classique à la fréquence de $1,2 \mathrm{~Hz}$. La figure 6 montre le spectre de relaxation obtenu à $1 \mathrm{~Hz}$ sur une éprouvette de Cuivre de haute pureté, après recuit in situ à $800^{\circ} \mathrm{C}$. On distingue deux pics l'un vers $400^{\circ} \mathrm{C}$ (L. T. P.) l'autre vers $700^{\circ} \mathrm{C}$ (H. T. P.).
Conclusion. - En conclusion les résultats obtenus grâce à ce pendule de flexion sont tout à fait comparables à ceux donnés par les pendules de torsion composés classiques. Le fond continu de l'ordre de $Q^{-1}=10^{-4}$, notamment, est en particulier du même ordre. On peut cependant remarquer que :

- L'exploitation des résultats est beaucoup plus facile que dans le cas du pendule de torsion composé.

- Il est possible d'utiliser des éprouvettes beaucoup plus rigides et de faire varier leur épaisseur de façon assez importante (de 0,5 à $2,5 \mathrm{~mm}$ ).

- On peut obtenir un rapport de fréquence élevé, de l'ordre de 10 , avec des éprouvettes rigides $(e=2,5 \mathrm{~mm})$ sans volant d'inertie prohibitif.

- Le montage des éprouvettes est particulièrement aisé ce qui rend l'emploi de l'appareil très commode.

- Cet appareil permet enfin de solliciter en flexion des éprouvettes dans un domaine de fréquence inférieur à celui généralement utilisé dans ce cas et donc de s'abstraire éventuellement de l'effet thermoélastique par un décalage en fréquence.

\section{Bibliographie}

[1] Chevenard (P.), Revue de Métallurgie, 1941, XXXVIII, 273.

[2] Collette (G.), C. R. Acad. Sci., Paris, 1958, 246, 2756.
[3] Ké (T. S.), Phys. Rev., 1947, 71, 533-546.

[4] Perez (J.), Guenin (G.), Gobin (P.), Mem. Sc. Rev. Met., 1968, 2, 135 . 